\title{
Determinants of Hospital Discharge Process: Experience from an Apex Tertiary Care Autonomous Institute of National Importance of Eastern India
}

\author{
Mukunda Chandra Sahoo ${ }^{1}$, Susmita Dora², Yatin Talwar ${ }^{3}$
}

\begin{abstract}
Background: The discharge of a patient is a time-consuming process comprising of clinical, financial, legal, and administrative and recordkeeping aspects that require proper execution. The discharge process is considered as an important quality indicator of the healthcare services of a hospital. Every hospital has its own discharge policy.

Objectives: To study the steps in the discharge process in a tertiary care teaching hospital and identify the causes of delays in in-patient discharges in major clinical wards.

Materials and methods: The study was conducted in four major clinical wards of AllMS, Bhubaneswar. The discharge process was recorded using a structured format that was distributed in all the wards. The patient satisfaction and impression regarding the discharged process were recorded using a questionnaire.

Results: The various steps identified in the discharge process were discharge summary writing, billing clearance, and patient leaving the hospital. Billing clearance contributed the maximum time from the total time taken for discharge followed by the discharge summary writing. Most of the patients rated their discharge process experience as above average and opined that the discharge process in the hospital was well organized. Conclusion: Discharging patients on time is a challenging task. With adequate manpower and proper patient counseling, the time taken for the process can be reduced. Improving the time taken for a discharge can improve the patient's satisfaction as well as effective bed management for the hospital.
\end{abstract}

Keywords: Discharge process, Discharge at tertiary care institute, Patient discharge, Patient satisfaction, Time taken for the discharge. International Journal of Research Foundation of Hospital and Healthcare Administration (2019): 10.5005/jp-journals-10035-1112

\section{INTRODUCTION}

An "inpatient" is a person who has been admitted to a hospital for purposes of receiving inpatient hospital services. ${ }^{1}$ A patient has to go through a set of stages during his/her stay in a hospital like admission, management, and discharge. ${ }^{2}$

As per National Accreditation Board for Hospitals and Healthcare providers (NABH), "Discharge is a process by which a patient is shifted out from the hospital with all concerned medical summaries ensuring stability." The discharge process starts after the approval of the treating physician and ends with the patient leaving the clinical ward. ${ }^{3}$ Two of the bottlenecks in hospital services are the admission and discharge processes. These processes can affect the quality of services of a hospital adversely (Davies and Macaulay). The discharge process is also a quality indicator for the quality of care and patient satisfaction as well. ${ }^{1}$ Delay in discharge of the patient also reduces bed availability of the hospital. Delay in discharge is bad for both hospitals and patients. It increases the cost from both the hospital and patient perspective. The increased the length of stay and also exposes the patient to hospital-acquired infections (Hendy et al., 2012). ${ }^{4}$ So, there is a requirement of effective strategies to effectively manage the discharge process. A standard time duration of 3 hours ( 180 minutes) is being suggested by the National Accreditation Board for Hospitals and Health Care Organizations.

According to Silva et al. (2014), the faulty operation processes are the major reasons for the delay and appropriate interventions can improve the process. ${ }^{5}$ The study was conducted in four clinical wards of a tertiary care teaching institute of national importance. The main reasons identified for the delay were waiting for the \begin{tabular}{l}
\hline 1,30partment of Hospital Administration, All India Institute of Medical \\
Sciences, Bhubaneswar, Odisha, India \\
2Department of Community and Family Medicine, All India Institute of \\
Medical Sciences, Bhubaneswar, Odisha, India \\
Corresponding Author: Mukunda Chandra Sahoo, Department \\
of Hospital Administration, All India Institute of Medical Sciences, \\
Bhubaneswar, Odisha, India, Phone: +91 9438884113, e-mail: \\
mcsahoo1@gmail.com \\
How to cite this article: Sahoo MC, Dora S, Talwar Y. Determinants of \\
Hospital Discharge Process: Experience from an Apex Tertiary Care \\
Autonomous Institute of National Importance of Eastern India. Int J \\
Res Foundation Hosp Healthc Adm 2019;7(2):55-57. \\
Source of support: Nil \\
Conflict of interest: None
\end{tabular}

test reports and delays in making clinical decisions and providing specialized consultation.

\section{Materials and Methods}

The present investigation was limited to AlIMS, Bhubaneswar. The study was conducted in four major clinical wards (medicine, pediatrics, obstetrics and gynecology, general surgery) of the hospital. All the inpatients who got discharged from the hospital in the month of June 2018 were considered for the study and those who did not give consent for the interview were excluded. However, all the patients consented for the interview and were 
included. A total of 250 patients were discharged during the study period. A structured proforma was used to record the break-up time of discharge and it was distributed in all the wards during this study period. The staff nurse of the concerned clinical wards were trained to fill the proforma. A self-administered questionnaire was distributed among a sample of 123 patients to record patient opinion and satisfaction for the discharge process. For statistical analysis, SPSS, IBM-20 was used.

\section{Results and Analysis}

The total number of discharges during the study period were 41, 35,28 , and 19 in obstetrics and gynecology, pediatrics, medicine, and surgery wards, respectively (Table 1).

The mean time taken for the discharge process was 195 minutes ( \pm 32 ). The longest discharge process was seen in patients where the average length of stay crossed more than 10 days. The time taken for patients of the gynecology department was significantly less than those of patients admitted under general surgery. This was primarily due to more residents in the department and also due to part preparation of discharge summaries a night before the day of planned discharge. The pediatrics department had the highest delay in discharges, primarily due to the center being a tertiary care facility that gets patients from a long distance.

This can also be attributed to the requests made by next of the kin to adjust discharge as per their convenience and travel schedules. As per the below-mentioned graph, more than $90 \%$ of discharge summaries were either prepared by the previous night or within 30 minutes of the advice of discharge being given (Fig. 1).

Figure 1 depicts the time taken for discharge summary preparation. It was observed that less than $10 \%$ of the discharge summaries were prepared after 30 minutes of the advice given for discharge.

Another attributable factor leading to delay in discharge was the time taken for billing clearance. It was observed that approximately one-third of the discharges were delayed by more than 1 hour due to the time consumed for billing clearance (Fig. 2).

Table 1: Distribution of total number of patient discharges according to various departments

\begin{tabular}{lll}
\hline S.no. & Department & Total discharges (\%) \\
\hline 1 & $\begin{array}{l}\text { Obstetrics and gyne- } \\
\text { cology }\end{array}$ & $41(33.33)$ \\
2 & Pediatrics & $35(28.45)$ \\
3 & Medicine & $28(22.76)$ \\
4 & Surgery & $19(15.44)$ \\
\hline
\end{tabular}

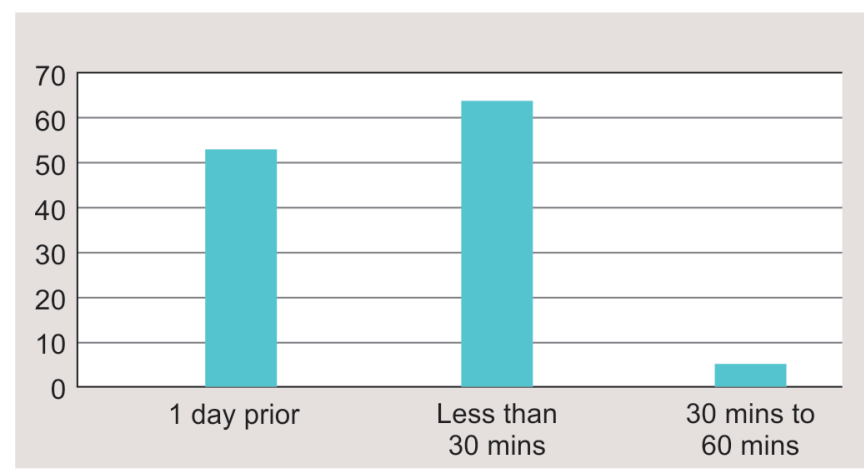

Fig. 1: Time taken for discharge summary preparation
The time taken for writing discharge summary, typing of discharge summary, and completion of billing was found to contribute immensely to the delay in total time taken for discharge. Except for the department of pediatrics, the time taken for the patient to leave the ward contributes only $10 \%$ of delays. The results obtained are found to be statistically significant with $p<0.05$. The billing process was mentioned as one of the longest steps in the discharge process that contributed around $50 \%$ of the total process. Next to it, discharge summary completion was mentioned as the second-longest process in the discharge process. Around $40 \%$ of the patients attributed their preference of time and date of discharge as a cause of delay in discharge.

On the evaluation of patient readiness for discharge, it was found that for general patients and all short procedures that had an approximated average length of stay, possible date of discharge at the time of their admission itself was intimated to the relatives and the patient. However, for super specialty cases and trauma cases, the average length of stay and possible time of discharge could not be intimated because of the presence of many variables. This was also a key reason for the preparedness of relatives toward the discharge process and hence leading to the relatives evacuating the bed earlier.

\section{Patient Satisfaction for the Discharge Process}

\section{Experience}

The majority of the patients rated the behavior of the hospital staff as good, which conciliates that they were satisfied with the behavior of the staff involved in the discharge process. Whereas for the experience of the discharge process, most of the patients rated the process as average, which also can hint that the fall of ratings was attributed to the delay in the billing process and other clearances.

\section{Findings and Recommendations}

Of the total patient's discharges, $2.5 \%$ (30) discharges had taken more than that of the standard time prescribed by the $\mathrm{NABH}$, i.e., 3 hours (180 minutes). The key recommendation to correct this deviation, where the time was found to be increased beyond the cutoff values (as prescribed by the NABH), can be to appoint a ward-based coordinator cum medical typist, who can coordinate and monitor the discharge process and discharges. Billing was the step that took the longest time in the discharge process followed by discharge summary completion as mentioned by the patients.

Hence, adequate staff deployment in the billing counters depending on the patient load can also be sought for. The billing time was found to be reduced by $10 \%$ in those wards having nursing trainee students. So, trainee students can be utilized for effective management of the discharge process, especially billing time.

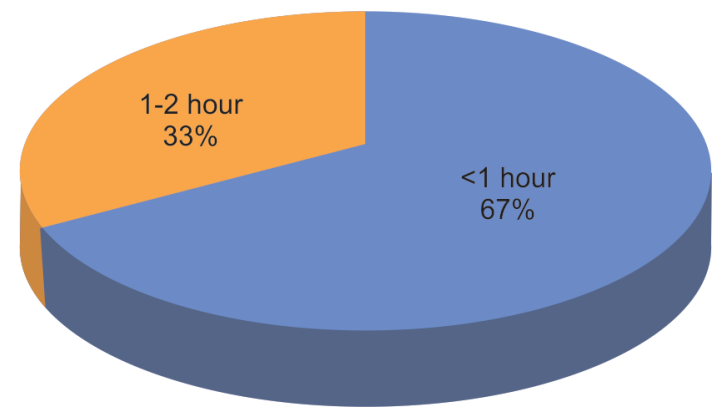

Fig. 2: Time taken for billing clearance 
Discharge summary preparation was another contributing factor for delay in the process of discharge. Training of the staff in the discharge process, especially the communication skills, for effective communication and coordination is required, which can eventually lead to a decrease in the delay of the discharge process.

It was also observed that many patients were not counseled for the prerequisites of the billing process. This increased the time taken for billing completion as the majority of the patients would not be ready with the billed amount during the billing process. A simplified solution to counsel the patients and relatives about their discharge date and prerequisites of the billing prior to the day of the discharge can lead to a reduction in time for the discharge. It was observed that the areas where patients and the attenders were made active participants of the discharge process, the delay in discharge was the lowest.

There is a requirement of regular feedback from the patients regarding the discharge process and corrective measures as per the feedback can substantially improve the process and reduce the turnaround time.

\section{Conclusion}

The discharge of a patient from hospital requires coordination at all the steps involved and also cooperation of the different staffs involved in the process. Discharge of a patient is an activity common to every hospital-small, large, community, teaching or nonteaching. Discharging the patient in a timely manner is a challenging task. Timely discharge not only increases satisfaction of patient and their relatives but also increases the inpatient bed turnover in the hospital. In this study, steps in the discharge process and the time involved were calculated and analyzed. It was found that the step in consuming most of the time of discharge was billing completion. With optimal staffs and counseling of the relatives at the time of discharge, the time for billing clearance can be reduced. The clearance charts and the hospital planning checklist must be regularly monitored to keep a track of all the delays and control them immediately. To successfully implement the discharge process, improvements in communication, doctors discharge order, discharge summary preparation, entry into electronic database, and analysis must be the methods to keep a record of the discharge process.

\section{References}

1. Sunil S, Sarala KS, Shilpa RG. Analysis of time taken for the discharge process in a selected tertiary care hospital. Int J Manag Appl Sci 2016;2(10):4-8. ISSN: 2394-7926.

2. Kumari JV. A study on time management of discharge and billing process in tertiary care teaching hospital. Elixir Int J 2012;52A: 11533-11535.

3. Ajami S, Ketabi S. An analysis of the average waiting time during the patient discharge process at Kashani Hospital in Esfahan, Iran: a case study. Health Information Management Journal 2007;36(2):37-42. ISSN 1833-3583.

4. Hendy $\mathrm{P}$, Patel $\mathrm{JH}$, Kordbacheh $\mathrm{T}$, et al. In-depth analysis of delays to patient discharge : a metropolitan teaching hospital experience. Clin Med (Northfield II). 2012;12(4):320-323.

5. Freitas $A$, Silva-Costa $T$, Lopes $F$, et al. Factors influencing hospital high length of stay outliers. BMC Health Serv Res 2012;12:265. 